\title{
Implicaciones de la ley estatutaria 1751 de 2015 sobre las entidades promotoras de salud del régimen contributivo*
}

\author{
Implications of statutory law of 2015 on 1751 health promotion entities contributory scheme
}

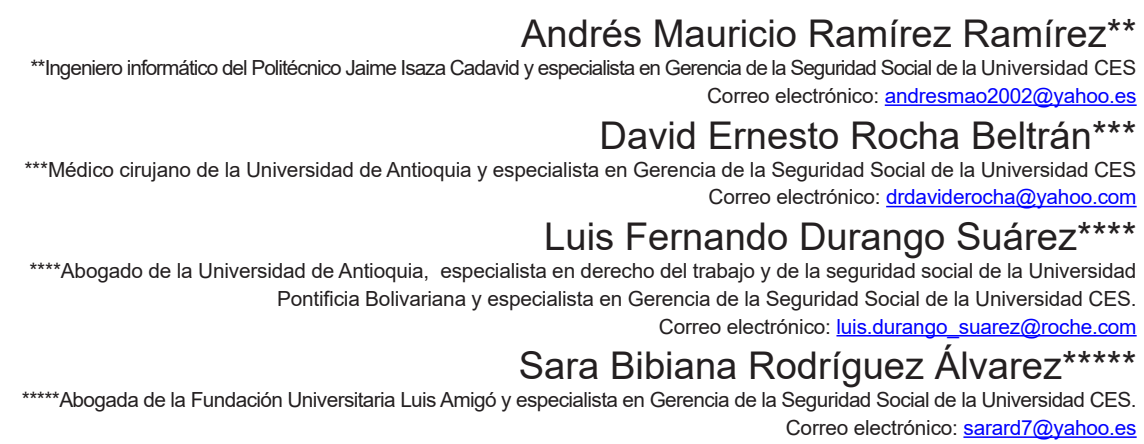

\section{Resumen}

La ley estatutaria 1751 de 2015 regula el derecho fundamental a la Salud, basada en unos principios y elementos esenciales que serán los encargados de impactar la prestación de los servicios y tecnologías en salud con el fin de asegurar una atención acorde a las necesidades de la población. Con esta ley, la cual prevé un período de transición de hasta dos años para algunos aspectos, se establecerán políticas que definan nuevos esquemas de beneficios y unos nuevos criterios en la prestación de los servicios, cambios que involucran a todos los actores del sistema y que requieren que estos adopten una nueva actitud frente al mismo, entiendan la salud como un concepto integral e integrador y comprendan que los determinantes de esta son dinámicos, cambiantes y requieren de su participación activa; que se conviertan en protagonistas de estos cambios y que hagan parte de la formulación de iniciativas tendientes a consolidar y garantizar el goce efectivo del derecho fundamental a la salud.

Palabras clave: Barreras de acceso, seguridad social, continuidad, servicios en salud, Ley Estatutaria, accesibilidad, integralidad, oportunidad, Constitución Política.

\begin{abstract}
The 1751 Statutory law of 2015 regulates the fundamental right to health, based on principles and essential elements that will be in charge of impacting the provision of health services and health technologies in order to ensure an attention according to the needs of the population. With this law, which foresees up to a two years transition period for some of the aspects, policies that define new schemes of benefits and criteria's on services provision will be establish, changes that will involve all the players in the system and that require that they adopt a new attitude towards them, understanding health as an integral and integrating concept and comprehend that the health determinants are dynamic, veering and require of their alive participation; and to become the leading actors of these changes, being part of the construction of new initiatives aiming to strengthen and ensure the effective enjoyment of the fundamental right to health.
\end{abstract}

Keywords: Barriers to entry, Social Security, continuity, Health Services, statutory Law, access, integrity, Opportunity, Constitution, autonomy.

* El artículo que se presenta a continuación es producto del trabajo de grado realizado para obtener el título de especialistas en el programa Gerencia de la Seguridad Social ofrecido por las facultades de Derecho y Medicina de la Universidad CES. 


\section{Introducción}

Desde sus orígenes, el sistema de aseguramiento de la salud en Colombia ha sido objeto de múltiples debates; de manera reciente, se ha declarado que dicho sistema se encuentra en crisis, razón por la cual se han realizado ingentes esfuerzos para superar los problemas financieros y solucionar las dificultades en cuanto a la oportunidad y calidad de los servicios. Así mismo, se ha hecho pública la necesidad latente de una reestructuración contundente del sistema, en vez de reformas reglamentarias, tal como lo evidencian las constantes noticias que dan cuenta de los casos de corrupción, la escasa reducción en las cifras de morbilidad y mortalidad en la población, la insuficiencia de la red pública asistencial y el inconformismo de los usuarios (Méndez, 2015).

Con el objeto de paliar la situación descrita, se promulgó la Ley Estatutaria 1751 de 2015, que regula el derecho fundamental a la salud, buscando solucionar los problemas de la atención relacionados con la calidad, oportunidad y el acceso a los servicios de salud. Esta ley ha buscado tener un mayor alcance al regular sobre los ámbitos de la práctica profesional en relación con la autonomía médica y la relación médicopaciente (Calderón, 2015), relación histórica que define la percepción de los usuarios acerca del servicio de salud que reciben.

El propósito de la norma es superar las barreras actuales del sistema que han repercutido en la prestación del servicio, con lo que se espera impactar positivamente la situación de salud de los colombianos; sin embargo, estos cambios implican una modificación en la forma en que los actores del sistema interactúan, incluso en la forma en que el talento humano en salud se relaciona con las instituciones del sistema. (Ley estatutaria 1751 de 2015).

Todos estos grandes cambios, sumados a las reformas en el financiamiento del Sistema General de Seguridad Social en Salud, representan importantes retos y adaptaciones que tendrán que asumir los actores del mismo, incluyendo a las Entidades Promotoras de Salud del régimen contributivo, quienes cuentan con un tiempo limitado para adaptarse a esta normatividad y marchar bajo las nuevas reglas de juego.

\section{De los principios del derecho fundamental a la salud}

La salud como derecho fundamental es un concepto relativamente nuevo para nuestro país, esto no siempre fue así, pues se consideraba un derecho de segunda generación o prestacional. Para que esto fuera una realidad han transcurrido una serie de cambios normativos, doctrinales y jurisprudenciales a lo largo de la historia; es así como en la década de los 80 en el Sistema Nacional de Salud, se establecieron unos principios básicos e incipientes que buscaban establecer un servicio público de salud, entre ellos se encontraban la universalidad, participación ciudadana y comunitaria, subsidiariedad, complementariedad e integración funcional (Ley 10 de 1990); estos principios buscaban mejorar el acceso a los servicios de salud de la población Colombiana; mas seguía dejando la salud como un derecho de segunda categoría.

Posteriormente con la Ley 100 de 1993 se dieron cambios influenciados por las tendencias neoliberales del contexto global (Gomez y Nieto, 2014); pero se hace un avance importante en cuanto a principios rectores del ahora llamado Sistema General de Seguridad Social Integral, se incluyen los principios de eficiencia, solidaridad, integralidad y unidad; estos sin embargo con un enfoque económico de la salud, pues se creó con dicha ley un "mercado regulado de salud" en donde las empresas y actores deben ser auto sostenibles y velar por el eficiente uso de los recursos; de esta forma la Salud aunque reconocido como bien "supremo" sigue estando supeditado al presupuesto y de esta manera se establecen unos planes de salud con coberturas definidas. 
Recientemente la Ley 1751 de 2015 (Ley Estatutaria en Salud) ha tomado varios elementos del Derecho para resaltar su naturaleza como derecho fundamental. De este modo, la Declaración de Derechos Humanos, el Pacto Internacional de Derechos Económicos, Sociales y Culturales, en especial la observación 14 y la Constitución Política de Colombia, han provisto las bases legales y constitucionales para instituir el derecho fundamental a la salud con base en sus características sustantivas, entre otras, que es inherente a la condición humana, es irrenunciable, que no prescribe y que es deber del Estado garantizarlo; para ello la citada norma incluye 18 elementos esenciales y principios que pretenden garantizar la prestación de los servicios de Salud de una manera que consoliden dicho derecho y lo conviertan en una realidad.

Para el presente trabajo, tomaremos cuatro principios de los incluidos recientemente y los analizaremos a la luz de la normatividad, de la jurisprudencia y de la evidencia actual así como el impacto que tienen y los cambios que en teoría tendrán sobre la prestación de los servicios de salud. Consideramos pertinente analizar estos principios por su relevancia en la materialización del derecho a la salud como derecho fundamental, su carácter integrador y porque en la realidad del sistema de salud Colombiano históricamente estos aspectos son los que más se ven vulnerados (Superintendencia Nacional de Salud, 2015) y no permiten el goce efectivo del derecho; finalmente analizaremos a la luz de los principios la problemática actual y lo que se espera con la entrada en vigor de la ley estatutaria, así como las implicaciones que esto tendrá para las aseguradoras.

\section{Principio de Continuidad, Integralidad, Oportunidad y accesibilidad.}

Estos principios han sidoreconocidos pornormas internacionales contenidas en tratados sobre derechos humanos ratificados por Colombia. De acuerdo con el concepto de bloque de constitucionalidad estos principios que hacen parte de los tratados con fuerza normativa similar a la de las normas constitucionales, consagran o definen derechos inherentes a la persona y permiten dar garantías para el derecho a la salud y así mismo su justiciabilidad e interpretaciones autorizadas para tomar decisiones respecto a esta.

Entre los tratados internacionales relacionados con el derecho a la Salud encontramos por ejemplo el PIDESC (Pacto Internacional de Derechos Económicos sociales y culturales) artículo 12 (Organización de las Naciones Unidas. Asamblea General., 1976), Declaración Universal de los Derechos Humanos artículo 25 (Organización de las Naciones Unidas, 1948), Protocolo de San Salvador (Organización de los Estados Americanos, 1988) Convenio de Ginebra y protocolos adicionales; todos estos instrumentos entre otros obligan a Colombia a adaptar su normatividad interna para cumplir con estos compromisos, es así como se establecen elementos esenciales y principios como los tratados en el presente artículo.

La Corte Constitucional ha sostenido que el derecho a la salud tiene naturaleza de derecho fundamental de manera autónoma; en este sentido la sentencia T-860 de 2003 menciona que la prestación de servicios de salud tiene un carácter vinculante para los entes encargados; en caso del desconocimiento de sus obligaciones se estaría en presencia de la violación de un derecho fundamental; cuya protección puede ser invocada de manera autónoma y directa.

Otras sentencias como la T-016 de 2007 protege el derecho a la salud como derecho fundamental, también la T-760 de 2008 vincula algunas características "principios" en la prestación de los servicios de salud, por último en la sentencia T-121 de 2015 la corte continúa ratificándolo como un derecho fundamental. 
Finalmente, el derecho a la salud se encuentra históricamente reconocido por la Constitución Política de Colombia en sus artículos 44,48 y 49 (Constitución Política, 1991).

\section{Continuidad}

El origen etimológico de la palabra continuidad proviene de "continuitas", se encuentra compuesta por las siguientes partes: El prefijo "con", que es equivalente a "junto". El verbo "tenere", que es sinónimo de "retener" o "dominar". El sufijo "uo", que se emplea para indicar relación. Es un término que se refiere al vínculo que mantienen aquellas cosas que están de alguna forma, en continuo. La palabra continuidad se define como la cualidad de no ser interrumpido, permanecer o hacer durar algo (Diccionario etimiológico, 2015).

Según Martín Zurro continuidad en salud es la: "Atención a lo largo de la vida de las personas, en sus distintos ámbitos (domicilio, escuela, trabajo, etc.) y en cualquier circunstancia (consulta en el centro de salud, urgencias, seguimiento hospitalario)" (Gálvez, 2003, p. 4). La continuidad busca evitar que se deje de prestar un servicio básico para todos los usuarios, que no se vulneren derechos fundamentales como el derecho a la vida.

La Ley 100 de 1993 no planteó la continuidad como principio, pero en el Decreto 3045 de 2013 se "dictan medidas para garantizar la continuidad en el aseguramiento... y establece que toda persona que ha ingresado al sistema de seguridad social en salud tiene vocación de permanencia y no debe, en principio, ser separado del mismo cuando esté en peligro su calidad de vida e integridad" (Ministerio de Salud y Protección Social, 2013)

La Corte ha sostenido que la continuidad en la prestación de servicios de salud, se le debe garantizar a los usuarios cuando inicien un tratamiento sin importar el estado de afiliación al sistema, es decir, los tratamientos médicos debe ser terminados hasta que los pacientes se recuperen o estabilicen, esto sin interrupciones que pongan en peligro los derechos fundamentales a la salud, integridad o a la dignidad (Sentencia T - 214 de 2013).

En la ley Estatutaria se define este principio como aquel en que, "las personas tienen derecho a recibir los servicios de salud de manera continua. Una vez la provisión de un servicio ha sido iniciada, este no podrá ser interrumpido por razones administrativas 0 económicas..."(Ley estatutaria 1751 de 2015).

Podemos destacar que aunque en la evolución normativa no ha estado siempre presente este principio, se ha incluido de diferentes maneras, buscando en todo momento brindar atención y una cobertura integral a todas las personas que lo requieran, que puedan contar con un servicio completo y eficiente de principio a fin. Es por esta razón que el Estado, las aseguradoras y las instituciones prestadoras de servicios de salud siempre deben trabajar de manera articulada para lograr este objetivo y que independientemente donde se presten los servicios o el tratamiento que se requiera, los usuarios no tengan barreras para la atención y se garantice su derecho fundamental a la salud.

\section{Integralidad}

El origen del término integralidad se deriva de integridad, de origen latino integrĭtas, hace hincapié en la particularidad de íntegro. Algo íntegro es una cosa que posee todas sus partes intactas (Diccionario etimiológico, 2015).

La integralidad, tiene varios sentidos, y uno de ellos consiste en realizar un conjunto articulado y continuo de acciones preventivas y curativas, individuales y colectivas, en los diferentes grados de complejidad del sistema, donde los servicios deben estar organizados para realizar una atención ampliada de las necesidades de la población a la que atienden (Kloh, et. al., 2014).

En nuestro Sistema de Seguridad Social el principio de integralidad ha sido postulado por 
la Corte Constitucional para las situaciones en las cuales, los servicios de salud requeridos son fraccionados o separados. Esta situación de fraccionamiento del servicio se debe por ejemplo al interés que tiene la entidad responsable en eludir un costo que a su juicio no le corresponde asumir (Sentencia T-760 de 2008).

Esto en contravía del Preámbulo de la ley 100 de 1993 en la cual nos indica que el sistema está diseñado para, "Gozar de una calidad de vida, mediante el cumplimiento progresivo de los planes y programas que el Estado y la sociedad desarrollen para proporcionar la cobertura integral de las contingencias" (Ley 100 de 1993).

Esta ley en su artículo $2^{\circ}$ define el principio de Integralidad como la cobertura de todas las contingencias que afectan la salud, la capacidad económica y en general las condiciones de vida de toda la población, y en su artículo 162 indica que "El plan obligatorio de salud es para todos los habitantes del territorio nacional, para la protección integral de las familias a la maternidad y enfermedad general, en las fases de promoción y fomento de la salud y la prevención, diagnóstico, tratamiento y rehabilitación para todas las patologías" (Ley 100 de 1993).

Este principio es ratificado por la Ley 1438 de 2011 dentro de la calidad de los servicios, así: "Los servicios de salud deberán atender las condiciones del paciente de acuerdo con la evidencia científica provisto de forma integral, segura y oportuna, mediante una atención humanizada" (Ley 1438 de 2011).

Finalmente, la Ley Estatutaria de Salud le dedica un artículo especial al principio de integralidad, cuya garantía también se orienta a asegurar la efectiva prestación de este servicio:

El artículo 8 de la Ley 1751 de 2015 establece que:
La integralidad. Los servicios y tecnologías de salud deberán ser suministrados de manera completa para prevenir, paliar o curar la enfermedad, con independencia del origen de la enfermedad o condición de salud, del sistema de provisión, cubrimiento o financiación definido por el legislador. No podrá fragmentarse la responsabilidad en la prestación de un servicio de salud específico en desmedro de la salud del usuario. En los casos en los que exista duda sobre el alcance de un servicio o tecnología de salud cubierto por el Estado, se entenderá que este comprende todos los elementos esenciales para lograr su objetivo médico respecto de la necesidad específica de salud diagnosticada. (Ley 1751 de 2015).

Es claro que las normas indican la obligación expresa de la integralidad en la atención a que tienen derecho las personas pertenecientes al sistema de seguridad social en salud, por tal motivo la atención integral en salud es una obligación forzosa de todos los entes encargados de la prestación del servicio público de salud.

En estos momentos con el fin de salvaguardar el derecho fundamental a la Salud el principio de integralidad ha sido uno de los criterios aplicados por la Corte Constitucional; las entidades que participan en el Sistema de Seguridad Social en Salud deben prestar un tratamiento integral a sus pacientes, con independencia de que existan prescripciones médicas que ordenen de manera concreta la prestación de un servicio específico. ${ }^{1}$

Por lo tanto, las personas vinculadas al Sistema General de Seguridad Social en Salud independiente del régimen al que pertenezcan, tienen el derecho a que las EPS les garantice

1 Este pronunciamiento de la Corte Constitucional es común en diferentes fallos a manera de ejemplo los siguientes: T-079 de 2000, T-133 de 2001, T-122 de 2001, T-136 de 2004, T-319 de 2003, T-1059 de 2006, T-830 de 2006, T-062 de 2006, T-760 de 2008, T-053 de 2009, T-574 de 2010. 
un servicio de salud adecuado, es decir, que satisfaga las necesidades de los usuarios en las diferentes fases, desde la promoción y prevención de enfermedades, hasta el tratamiento y rehabilitación de la enfermedad y con la posterior recuperación; por lo que debe incluir todo el cuidado, suministro de medicamentos, cirugías, exámenes de diagnóstico, tratamientos de rehabilitación y todo aquello que el médico tratante considere necesario para restablecer la salud del paciente o para aminorar sus dolencias y pueda llevar una vida en condiciones dignas (Sentencia T-179, 2000), (Sentencia T-988, 2003), Sentencia T- 568, 2007), (Sentencia T-604, 2008), (Sentencia T-136, 2004), (Sentencia T-518, 2006), (Sentencia T-657, 2008), (Sentencia T-760, 2008).

\section{Oportunidad}

El término oportunidad viene del latín oportunitas que a su vez proviene de ob que significa "hacia" o de op "antes" y portus que significa "puerto"; este término se usaba en el mar para referirse a las condiciones de viento, marea y corriente para avanzar hacia el puerto o destino. De esta forma una embarcación se encontraba en estado de opportunitas cuando su capitán había decidido a donde ir y sabía cómo llegar en "el tiempo justo". El concepto como tal se define según la Real Academia de la lengua Española (RAE) como el momento o circunstancia oportunos o convenientes para algo, a su vez oportuno la RAE lo define como lo que se hace o sucede en tiempo a propósito y cuando conviene. (Diccionario etimiológico, 2015)

El principio de oportunidad como concepto en la prestación de servicios de salud se puede entender como la capacidad de satisfacer la necesidad de salud de la persona en el momento preciso en que requiere dicho servicio (Duran, 2004). De hecho el Ministerio de Salud de Colombia lo tiene dentro de uno de los atributos de la calidad de la atención en salud y amplía el concepto entendiéndolo como la posibilidad que tiene el usuario de obtener los servicios que requiere sin que se presenten retrasos que pongan en riesgo su vida o su salud (Ministerio de Salud, 2015).

En nuestro sistema de Salud se habló de dicho principio propiamente hasta la ley 100 de 1993, artículo 153 y numeral 3 donde se nombró como atributo en la garantía de integralidad de la atención (Ley 100, 1993); posteriormente en el decreto 2174 de 1996 lo incluye como principio en el Sistema Obligatorio de garantía de calidad (Ministerio de Salud y Protección Social, 1996), no obstante quien ha usado el principio y lo ha hecho prevalecer en sus fallos es la Corte Constitucional, quien en varias sentencias cita este principio para fundamentalizar el derecho a la salud y lo define como la prestación del servicio en el momento que corresponde para la recuperación satisfactoria del estado de salud (Sentencia T-745 de 2013), y últimamente fue incluido en la ley estatutaria de salud.

En este aspecto la oportunidad juega un papel esencial a la hora de materializar la salud como un derecho, ya que este bien "supremo" se encuentra necesariamente ligado al factor "tiempo" y "espacio". En la triada epidemiológica (persona-tiempo-espacio) (Alegret, et. al., 2008), el patrón tiempo y espacio son esenciales para determinar la forma en que cursará el proceso salud-enfermedad; es así como una intervención o una omisión en la línea tiempo puede ser decisiva para que la persona empeore o mejore su condición de salud, e incluso entre en una serie de eventos desencadenantes que podrían llevar a la muerte.

En los últimos tiempos se habla de la doctrina de la pérdida de oportunidad o pérdida de chance, citada en el ámbito probatorio jurisprudencial del derecho de daños (en especial en la responsabilidad médica), en el campo de la salud se entiende como "el empeoramiento del pronóstico de una enfermedad como consecuencia de la demora excesiva o 
inadecuada en su estudio, diagnóstico y tratamiento" (De la Calle, 2006) de acuerdo con esta doctrina, si se demostrara que una intervención en salud hubiera podido evitar el daño procedería una indemnización, si bien menor que cuando existe una mala práctica.

Es por eso que la "oportunidad" se convierte en un elemento esencial para la materialización del derecho a la salud; esta oportunidad es decisiva en todos los niveles de aplicación de las actividades en salud desde la promoción y la prevención hasta la rehabilitación y prevención de secuelas o discapacidad. Es claro que hay actividades del proceso de atención en salud como el triage o clasificación de urgencias, la atención inicial de urgencias (Morales, 2008) y el proceso diagnostico-terapéutico en estados agudos de quebrantamiento de la salud en donde es posible establecer la causa-efecto de fallas en el elemento de oportunidad; sin embargo hay otras en que no es fácil hacerlo como por ejemplo en la promoción de la salud; pues la falta de oportunidad en esta actividad no se verá a corto sino a mediano y largo plazo, sin ser menos importante o sin dejar de generar un impacto notable y decisivo para la salud colectiva y el desarrollo de esa sociedad.

Actualmente la oportunidad en salud en la práctica se entiende como el tiempo de espera o la "demora" en la prestación del servicio de salud de cara al usuario; sin embargo la ley estatutaria al elevar la salud al nivel de derecho fundamental y al incluir el elemento oportunidad dentro de sus pilares, hace que el concepto sea mucho más amplio y que esté inmerso en todos los momentos que pueden definir el curso salud-enfermedad de un individuo e incluso de una comunidad, ahora la oportunidad se definirá a cada instante, en donde una acción que promueva o facilite ambientes o acciones que mejoren la salud se pueda llevar a cabo.

Para el sistema representa un reto, no solo de recursos (humanos, técnicos, tecnológicos) si no de identificar los momentos en el espacio- tiempo en que puede intervenir impactando de manera positiva los indicadores de salud de la población y del individuo en particular, también representa una oportunidad de hacer una medicina mucho más preventiva y menos morbicentrista, logrando también la consecución de logros en materia de sostenibilidad y eficacia en la ejecución de los recursos.

\section{Accesibilidad}

Para la Real Academia de la Lengua Española, el término accesibilidad denota la cualidad de accesible, es decir, que es de fácil acceso o trato, de fácil comprensión o inteligible ("accesibilidad", 2015). En el contexto sanitario, se refiere a la forma en que los servicios de salud se acercan a la población. Bajo esta óptica, el acceso a la salud constituye un indicador de desarrollo social (Comes, et. al., 2007) lo que en otras palabras implica acortar las brechas entre los ciudadanos y sus sistemas de gobierno y especialmente coartar las determinantes sociales de la salud que a modo de barreras de acceso a los servicios, impiden que las personas puedan recibir un servicio de salud de calidad, eficiente y equitativo.

La perspectiva de análisis que se emplee para entender el concepto de accesibilidad, permite visualizar las diferentes barreras de acceso a la salud. Por ejemplo, para Comes y colaboradoras, las barreras vistas desde la oferta, son de naturaleza geográfica, económica, administrativa y cultural. Para estas autoras, el problema de definir la accesibilidad desde la oferta, es que solapa la participación de las personas como constructores de la accesibilidad, es decir, incluir los discursos, las prácticas en relación con el cuidado de la vida y la salud como estrategia para mejorar el acceso y superar los encuentros y desencuentros entre la población y los servicios de salud (Comes, et. al., 2007).

Tovar-Cuevas y Arrivillaga han señalado que a pesar de las reformas en el Sistema de Salud Colombiano, aún persisten barreras de acceso, 
pues aún hay datos de población sin cobertura en salud, se mantiene una insuficiente distribución de los servicios, hay datos de corrupción y son evidentes las fallas en la atención a la salud pública (Tovar, at. al., 2014).

En Colombia, el concepto de acceso a los servicios de salud se ha intentado explicar operativamente, es decir, como la puerta de entrada al Sistema de Salud que se caracteriza por ser restringida y ajustada a datos e indicadores de cobertura y aseguramiento, por ejemplo, el número de camas hospitalarias por 1000 habitantes, la tasa de médicos por habitantes, el número de consultas de urgencias por cada 10 consultas externas en la red pública, etc. Por otra parte, la base de datos de afiliados del Ministerio de Salud que registra datos sobre el régimen de afiliación, muestra inequidades en el acceso, en las diferentes regiones del país, pues aún hay una proporción importante de la población sin cobertura en salud y prevalece la insuficiente distribución de recursos para la salud entre las zonas urbanas y rurales. Esto indica que a pesar de las reformas estructurales de que ha sido objeto el Sistema, las cifras no muestran las dificultades y barreras que efectivamente deben enfrentar los usuarios cuando buscan los servicios de salud.

A diferencia de las legislaciones que han precedido a la Ley Estatutaria, no se había hecho alusión directa al concepto de accesibilidad. En el afán de paliar las situaciones derivadas de las fallas estructurales del sistema sanitario, la Corte Constitucional ha proferido una serie de sentencias de tutela mediante las que ha buscado proteger el derecho a la salud, con base en los principios de integralidad y continuidad, y por ende de accesibilidad, como esencia del servicio público de la salud, con una visión que trasciende la enfermedad y los intereses económicos prevalentes (Vélez, 2012).

En el contexto de la Ley Estatutaria en Salud, la accesibilidad es entendida como la posibilidad de que los servicios y tecnologías puedan ser accesibles a toda la población en condiciones de equidad y con base en las características culturales individuales y colectivas. Bajo este principio rector, el concepto alude a la no discriminación, la accesibilidad física, la asequibilidad económica y el acceso a la información.

Así las cosas, se supera la perspectiva de la oferta de los servicios de salud derivada de la lógica del mercado y se enfoca en los determinantes sociales del estado de salud, mediante los que el Estado asume la responsabilidad de adoptar políticas encaminadas a disminuir las desigualdades que afectan el goce del derecho fundamental a la salud, y promueven el mejoramiento de las condiciones de salud, buscan prevenir la enfermedad y mejorar la calidad de vida de la población colombiana.

La base de datos única de afiliados del Ministerio de Salud aporta datos sobre el régimen de afiliación, no obstante, muestra inequidades en el acceso entre regiones de Colombia, los problemas estructurales del sistema siguen sin resolverse, las barreras de acceso se han mantenido. Persiste la presencia de población sin cobertura en salud (concepto que difiere de acceso real), se mantiene la inadecuada e insuficiente distribución de los servicios tato en las zonas rurales como en las urbanas.

La realidad del sistema en la prestación de los servicios de salud.

La ley 1751 de 2015 recoge varios elementos, principios y aspectos relacionados con la prestación de los servicios de salud con el fin de clarificar, unificar y validar conceptos clave que permitan la materialización del derecho a la salud, ahora reconocido oficialmente como fundamental.

En este sentido, abarca desde principios y elementos esenciales hasta aspectos del día a día en la prestación de los servicios como la 
atención de urgencias, los planes de beneficios e incluso aspectos que tienen que ver con la relación médico-paciente dándole un énfasis especial a aspectos como la autonomía médica y participación en salud.

En la actualidad hay varios temas críticos en relación a la forma en que se prestan los servicios de salud y aspectos que determinan la manera en que los usuarios interactúan con los prestadores de servicios, lo que define para fines prácticos si se materializa o no el derecho, bajo qué condiciones lo hacen y finalmente tienen un impacto real en la morbimortalidad de la población en lo relacionado con uno de los determinantes de la salud (Sistema sanitario).

En este orden de ideas es apropiado analizar la problemática actual de la prestación de servicios, como la atención de urgencias ahora priorizadas en la ley estatutaria, los planes de beneficios (la filosofía en que deberán ser diseñados e implementados) y la relación entre el médico y el paciente, ya que se convierten en aspectos claves para el goce efectivo del derecho y la aplicación de los principios bajo análisis en el presente artículo.

\section{Los planes de beneficio y la prestación de los servicios de salud}

Un elemento fundamental del Sistema General de Seguridad Social en Salud en Colombia es el Plan de beneficios, que corresponde al paquete de servicios básicos en las áreas de recuperación de la salud, prevención de la enfermedad y cubrimiento de ingresos de manera transitoria, este es una derivación de la cobertura de servicios que brindaba el Instituto de Seguros Sociales a sus afiliados.

En desarrollo de los artículos 48 y 49 de la Constitución política, la Ley 100 de 1993 prevé la creación de las condiciones para que tanto los afiliados al régimen subsidiado de salud como los afiliados al régimen contributivo de salud puedan acceder a un plan de beneficios denominado POS (Plan Obligatorio de Salud), el cual:
“(...) permitirá la protección integral de las familias a la maternidad y enfermedad general, en las fases de promoción y fomento de la salud y la prevención, diagnóstico, tratamiento y rehabilitación para todas las patologías, según la intensidad de uso y los niveles de atención y complejidad que se definan" (Artículo 162, inciso 1 de la Ley 100 de 1993).

La Ley 100 de 1993 no estableció plazos para la actualización. Al respecto, el parágrafo 2 del artículo 162 de la mencionada ley señala lo siguiente:

Los servicios de salud incluidos en el Plan Obligatorio de Salud serán actualizados por el Consejo Nacional de Seguridad Social en Salud, de acuerdo con los cambios en la estructura demográfica de la población, el perfil epidemiológico nacional, la tecnología apropiada disponible en el país y las condiciones financieras del sistema.

Esta situación fue asumida en un primer momento por el artículo 25 de la Ley 1438 de 2011, pues determinó que el Plan de Beneficios debería actualizarse integralmente una vez cada dos años, atendiendo a los cambios en el perfil epidemiológico, la carga de la enfermedad de la población, la disponibilidad de recursos, el equilibrio y los medicamentos extraordinarios no explícitos en el Plan de Beneficios (Ley 1438 de 2011).

Hoy la ley 1751 de 2015 señala que la ampliación del plan de beneficios debe ser progresiva, indica que durante los dos años siguientes a su expedición, el Gobierno Nacional y el Congreso de la República deberán establecer las políticas para la definición de un nuevo esquema de beneficios, incluido el mecanismo para realizar las exclusiones según los criterios establecidos por la misma ley (Ley 1751 de 2015).

La regulación de lo que debe contener el plan de beneficios generó gran incertidumbre en los actores del sistema, pues al no determinar 
un mecanismo específico, ni unos criterios de interpretación para su actualización terminó vulnerando flagrantemente el Derecho Fundamental a la Salud, ya que este implica el acceso efectivo a los servicios médicos que requiera una persona para conservar su estado de salud, cuando se encuentre comprometida su vida, su dignidad o su integridad personal, en condiciones de "oportunidad, continuidad, eficiencia y calidad," (Sentencia T-859 de 2003).

Cabe destacar entonces el pronunciamiento de la Corte Constitucional sobre los problemas recurrentes que ocasiona una lista taxativa de beneficios (POS positivo) ${ }^{2}$ (Giedion, et. al, 2014) que han quedado desactualizados por el paso del tiempo generando barreras al acceso, demoras en la atención, entrega de tratamientos incompletos e incluso la no entrega de los mismos; situaciones que vulneran el derecho fundamental a la salud de los usuarios.

En la sentencia T-025 de 2006 se consideró que la desactualización del POS afectaba los derechos fundamentales de los usuarios, se evidenció que el paso del tiempo impacta directamente la vigencia del plan de beneficios perjudicando a quien requiere de procedimientos no incluidos en el plan vigente, a tal punto de desconocer sus derechos constitucionales fundamentales a la vida, a la integridad personal y a la dignidad (Sentencia T-025 de 2006).

La desactualización del plan de beneficios por cerca de 10 años fue uno de los agravantes del sistema de salud, y las actualizaciones recientes no han sido suficientes para mejorar la percepción que se tiene de dicho plan $^{3}$ (El Tiempo, Redacción, 2012). Es claro 2 Según la División de Protección Social y Salud del Banco Interamericano
de Desarrollo el plan de beneficios positivo hace referencia a los
medicamentos, insumos, procedimientos y servicios que se definen en
un listado específico de lo que el sistema financia. Véase en: Banco
Interamericano de Desarrollo. Planes de beneficios en salud de América
Latina. Una comparación regional. Disponible en: https://publications.iadb.
org/bitstream/handle/11319/6484/Planes_de beneficios_en_salud_de
America Latina.pdf?sequence=4
3 Según el Primer Estudio de Percepción sobre el sistema de salud.
Realizado por DATEXCO para el Instituto de Altos Estudios en Salud, adscrito a la Asociación Colombiana de Sociedades Científicas. Véase que la desactualización del plan agrava el problema de las prestaciones, pero aún estando perfectamente actualizado tendrá inevitablemente servicios excluidos, más aún dados los avances médicos y la nueva tecnología que se desarrolla alrededor del mundo y que llega continuamente al país.

Esta situación deja usuarios con los mismos problemas, pues no encuentran diferencias con los cambios, ya que por lo general sus tratamientos siguen estando excluidos de los planes actualizados. La demora en citas y entrega de medicamentos tampoco mejora y las actualizaciones a la que es sometido el plan de beneficios no se perciben ya que los medicamentos y exámenes siguen siendo negados; todo esto sumado a la falta de calidad en la atención, demoras en el acceso a los médicos especialistas, condiciones que impiden que la tutela deje de ser la herramienta que tienen los usuarios para lograr la protección del su derecho fundamental a la Salud.

Como vemos el principal problema identificado de los planes de beneficios actuales es que se convierte en una lista cerrada de servicios, medicamentos e insumos que inevitablemente con el paso del tiempo y el avance científico médico y tecnológico se desactualiza y no cumple con el objetivo de mantener, preservar y promocionar la salud con criterios de racionalidad y costo-efectividad, esto a la postre convierte a la salud no en un derecho de primera generación, sino en un derecho prestacional que se supedita al presupuesto, voluntad y opinión de un grupo de personas que define "que entra y que no entra" en la lista y que finalmente obliga al usuario a usar mecanismos que garanticen la protección especial y amparo del derecho fundamental.

Por tal motivo y previendo la necesidad de superar esta falencia, la Ley Estatutaria de

en: El Tiempo, Redacción,. (2012). El 40\% de pacientes está inconforme con los servicios de salud Estudio de percepción sobre el sistema halló que siguen las demoras en asignación de citas. El Tiempo. Disponible en: http://www.eltiempo.com/archivo/documento/CMS-12421628 
Salud introduce un cambio estructural en cuanto al plan de beneficios; así mismo la última norma expedida para regular dicho plan, la Resolución 5592 de 2015, busca resolver provisionalmente el problema de actualización que sufre hasta la entrada en vigor de la Ley en mención (Ministerio de Salud y Protección Social, 2015).

Otro aspecto tiene que ver con el ámbito de la autonomía médica; pues se encuentra necesariamente ligada al tema de la prestación de los servicios; ya que la relación médico paciente se convierte en el "momento de verdad" de los servicios de salud y de dicha relación se define una serie de prestaciones con el fin de promocionar la salud y prevenir la enfermedad en todos sus niveles.

La autonomía se define como la facultad de la persona o la entidad para obrar según su criterio, con independencia de la opinión o el deseo de otros, basada en su propio sistema de valores y principios (Gracia, 1998); así mismo la Asociación Médica Mundial en la 39ª asamblea que se desarrolló en Madrid mencionó sobre la autonomía médica lo siguiente:

"El elemento principal de la autonomía profesional es la garantía de que el médico pueda emitir con toda libertad su opinión profesional con respecto a la atención y tratamiento de sus pacientes..." (Asociación Médica Mundial, 1987); en conclusión la autonomía medica se puede definir como "el actuar médico de manera personal, sin ninguna coacción interna o externa" (Cuasialpud, 2013).

Esta autonomía médica se basa en una relación de mutua reciprocidad y beneficio entre el médico y la sociedad y así quedó consignado en el Código de ética médica de la Asociación Americana de Medicina (AMA), publicado en 1847; esta propuso que el médico asumiera una serie de obligaciones frente a la comunidad; mientras esta última, a cambio, le otorga a la profesión una serie de prerrogativas especiales, entre las que sobresale la libertad para establecer sus propios estándares (Gualtero, 2007).; es decir le confiere autonomía al médico.

En Colombia a partir de la ley 100 de 1993 en donde se creó el mercado regulado de salud, el quehacer médico y la relación médicopaciente se comenzó a ver afectada por la intervención de un "tercero"; pues ya no era una relación bilateral sino que apareció la figura del "pagador" quien fiscalizaría los procedimientos, intervenciones y actuar médico que generaban costo para el sistema pero que se definían de acuerdo con la particularidad de la persona (paciente) y su problema de salud específico.

Con la implementación del Sistema de Seguridad social en salud; vinieron una serie de cambios normativos que buscaban la eficiencia y eficacia en el uso de los recursos, pero que desconocieron esa autonomía médica que caracterizó el ejercicio de la profesión por siglos; por ejemplo el Decreto 131 de 2010 (emergencia social) que fue declarado inexequible por la Corte Constitucional, creó organismos de vigilancia que buscaban el "control del gasto" mediante la auditoria y mediante la emisión de directrices de cara al gremio médico; no obstante estos comités o entes no estaban conformados en su totalidad por médicos sino por representantes de la cartera del gobierno con voz y voto lo que incumplía el principio de "autorregulación".

Incluso este decreto llegó a proponer sanciones económicas a los médicos que no se apegaran a dichas directrices incluyendo sanciones como estas:

\footnotetext{
"Sanciones pecuniarias. Cuando el profesional se aparte sin justificación aceptable de una recomendación incluida en un estándar adoptado por su respectiva profesión y con ello ocasione un daño económico al Sistema General de Seguridad Social en Salud, incurrirá en una falta que será sancionada con una
} 
multa entre 10 y 50 SMMLV." (Ministerio de Salud y Protección Social, 2010).

En la actualidad la práctica médica se encuentra sujeta a varias condiciones que deben ser cumplidas por los médicos al momento de recomendar a sus pacientes terapias o tratamientos (incluyendo estudios y demás), por ejemplo:

- Tiempo de consulta menor de 20 minutos

- Restricción en ciertas pruebas básicas de laboratorio

- Restricción en la formulación de ciertos medicamentos

- Restricción en la remisión a especialidades u otros niveles de atención.

De hecho en un artículo reciente publicado por una revista de opinión de circulación nacional se evidenció prácticas tendientes a la reducción del gasto pero que presionan y violan flagrantemente la autonomía del médico tratante; por ejemplo: Notificación periódica a los médicos de los gastos que generan sus decisiones, listas públicas con nombres de los médicos que más ordenan procedimientos o estudios, auditorias en "vivo" para procedimientos de baja complejidad y de bajo costo, notificaciones y alertas electrónicas para pacientes que superen un "tope establecido" en estudios en un periodo de tiempo y otras más (Semana. Redacción, 2014).

En cuanto a la autonomía la Constitución Política de Colombia la consagra en sus artículos 13, 16 y 18 donde relaciona dicha autonomía con el desarrollo de la libre personalidad y la libertad con las que nacen los ciudadanos (Const., 1991); de la misma forma la ley 23 de 1981 establece la autonomía del paciente y en su artículo 4 deja a criterio del paciente la elección del médico y la elección del tratamiento que quiera aceptar, así mismo el articulo 8 y el articulo 15 deja en potestad del paciente aceptar o no las alternativas presentadas por el médico y prescindir incluso de sus servicios (Ley 23, 1981); así mismo la resolución 13437 de 1991 del Ministerio de salud incorpora el decálogo de los derechos del paciente (Ministerio de Salud, 1991)

En cuanto a la autonomía médica la corte constitucional en sentencia T-823 de 2002 estimó que:

"Por regla general, resulta que no puede ni obligarse al paciente a seguir la prescripción propuesta por el médico en contra de su voluntad $y$, por ende, desconociendo su consentimiento idóneo, ni ordenarse al médico a actuar clínicamente en contra de los postulados de su profesión" (Sentencia T-823, 2002).

Así mismo la sentencia T-401 de 1994 dijo:
"Conceder una tutela para ordenar al médico que modifique un tratamiento normalmente admitido en el medio científico representa una indebida intromisión del juez en campos que desconoce $y$, lejos de proteger los derechos del paciente, se corre el peligro de afectarlos por ignorancia, quebrantando de paso el derecho del galeno a que se respete su autonomía profesional..." (Sentencia T-401 de 1994)

La autonomía del médico ha pasado por varias etapas durante la historia del sistema de Salud en Colombia; paso de ser una realidad cuando la medicina se ejercía de una forma liberal e independiente, a ser relativo en la medida en que se institucionalizó la prestación de los servicios médicos y se instituyeron guías o protocolos de atención que permitieron comparar el actuar médico "particular" contra los conceptos consensuados de agrupaciones científicas médicas; esto no afectó en forma significativa la forma de ejercer la práctica médica, solo hasta cuando se usaron estas guías y protocolos para juzgar y evaluar el actuar médico (auditoría) y con ello negar lo prescrito por el médico o 
sugerir conductas alternativas a la propuesta por el médico tratante.

Esta situación ha traído varios problemas de cara a la prestación de los servicios, pues lo que el medico prescriba o formula a su paciente no es seguro que le sea suministrado y tiene que pasar por controles que el sistema ha establecido como por ejemplo procesos de autorización por parte de las EPS (que en ocasiones eran complejos y demorados), los CTC (Comités Técnico Científicos) diseñados para prescripciones que se salieran del plan de beneficios; cuando estos servicios son negados, actualmente los usuarios acuden al mecanismo de tutela solicitando la protección de un derecho fundamental.

Es así que 2 elementos esenciales de la prestación de los servicios (el plan de beneficios y la autonomía médica) se convierten en factores clave en la materialización del derecho a la salud; pues de ellos depende la continuidad en la prestación de los servicios y la oportunidad en que estos son suministrados.

\section{El principio de continuidad y oportunidad en la prestación de los servicios de salud}

Este principio que se define brevemente como la propiedad de no ser interrumpido, permanecer o conectarse es uno de los más afectados con la problemática actual; pues la principal queja de los usuarios es la no entrega de los medicamentos formulados por su médico (por no estar incluido en el plan de beneficios o porque un comité considera que no está indicado "relacionado con la autonomía"); esto hace que el proceso de salud-enfermedad que por definición es un continuo (Martinez, A. \& Rubio, 1999), no reciba la atención sanitaria que requiere y por lo tanto el proceso de morbilidad en muchos casos no se impacta en la forma esperada, o se hace de forma tardía (inoportunamente) lo que ocasiona la aparición de complicaciones, secuelas y deterioro de la calidad de vida llegando incluso hasta la muerte de los usuarios.

\section{La prestación de servicios de Urgencias}

La palabra urgencia (“Urgencia”, 2015) viene del latín urgentǐa y hace referencia a la cualidad de urgente o que urge, apremia o requiere de pronta atención. Según la Organización Mundial de la salud, una urgencia "es la aparición fortuita de un problema de etiología diversa y de gravedad variable, que genera la conciencia de una necesidad eminente de atención por parte del sujeto o de sus allegados" (Organización Mundial de la Salud, 2010) a diferencia de una emergencia que es una situación en la que está en peligro la vida de la persona o la función de algún órgano de manera inmediata.

La Ley 100 de 1993 habla de cómo debe ser la atención inicial de urgencias, y en su artículo 168 lo define de la siguiente manera "La atención inicial de urgencias debe ser prestada en forma obligatoria por todas las entidades públicas y privadas que presten servicios de salud, a todas las personas, independientemente de la capacidad de pago. Su prestación no requiere contrato ni orden previa (Ley 100 de 1993).

La Resolución 249 de 1998 define el concepto de urgencia en su artículo $1^{\circ}$ :

"La alteración de la integridad física y/o mental de una persona, causada por un trauma o por una enfermedad de cualquier etiología que genere una demanda de atención médica inmediata y efectiva, tendiente a disminuir los riesgos de invalidez o muerte" (Secretaria Distrital de Salud de Bogotá, 1998).

El modelo de salud colombiano ha llevado que el proceso en los servicios de urgencias implemente un sistema de atención en el cual se le dé prioridad los pacientes según la gravedad que estos presentan, es por esto que se hace necesario emplear un sistema de clasificación llamado triage donde se busca que los pacientes sean atendidos no por el orden de llegada sino por la clasificación de sus síntomas y donde la evaluación de estos permita evidenciar 
la verdadera gravedad de su condición de salud y brindarles una atención oportuna. La adopción de estos sistemas de clasificación ha surgido del interés de evitar demoras en la atención, garantizar una rápida valoración de salud, disminuir los niveles de ansiedad en los pacientes y sus familias y garantizar una adecuada gestión de los recursos físicos y humanos para el manejo de los problemas de los pacientes, cuya complejidad y costos han ido aumentando.

En su momento, la Ley 100 de 1993 señaló en el Régimen de Beneficios del Plan Obligatorio de Salud, la necesidad de que las Entidades Promotoras de Salud, establecieran un sistema de referencia y contrarreferencia para que el acceso a los servicios de alta complejidad fuese canalizado desde el primer nivel de atención, excepto para las atenciones de urgencias.

Con base en el marco legal expuesto, el sistema de referencia y contrarreferencia se define como el conjunto de procesos, procedimientos y actividades técnicas y administrativas que permiten prestar adecuadamente los servicios de salud a los pacientes, garantizando la calidad, accesibilidad, oportunidad, continuidad e integralidad de los servicios, en función de la organización de la red de prestación de servicios definida por la entidad responsable del pago.

La palabra triage proviene del término francés "trier" que significa seleccionar, clasificar o elegir. Dicho término aplicado en el contexto de las instituciones sanitarias, hace referencia a los procedimientos desarrollados por el personal de salud para clasificar a los pacientes con base en su gravedad con el propósito de asignar la prioridad de la atención, y a partir de allí, tomar las decisiones clínicas necesarias para instaurar medidas terapéuticas o derivar a los sitios que puedan brindar la mejor atención posible (González, et. al., 2012)

En el contexto colombiano, el triage de los pacientes es una actividad que se ha venido llevando a cabo en las salas de Urgencias, a manos de profesionales en Medicina o Enfermería, o incluso personal técnico auxiliar, a pesar de que la norma que lo reglamenta indica hacerlo de otra manera.

En el año 1990, el artículo 2 de la Ley 10 por la que se reorganizó el Sistema Nacional de Salud, señaló que todas las instituciones que prestaran servicios de salud, estaban obligadas a proporcionar atención inicial de urgencias, independientemente de la capacidad socioeconómica del solicitante (Ley 10 de 1990). Posteriormente, la Ley 100 de 1993, en los artículos 167 y 168, también definió la atención inicial en salud de urgencia, hoy denominada atención de urgencias (Ley 100 de 1993). La implementación y desarrollo de la Ley 100 de 1993, impactó en las formas de prestación de servicios y en las condiciones de salud de las personas, al punto de privilegiar la atención hospitalaria especializada y limitar las actividades de promoción de la salud y prevención de la enfermedad.

Estos cambios derivados de la reforma sanitaria, modificaron las dinámicas institucionales, de manera que ante la alta afluencia de pacientes a las salas de urgencias se precisó la incorporación de algún sistema para la clasificación de los pacientes, para lo que buscaron hacer adaptaciones de los sistemas utilizados a nivel mundial, no obstante, sin un marco legal que soportara la estrategia o que sirviera de base jurídica para avalar las decisiones clínicas.

Esto trajo consigo una problemática ya que cada institución comenzó a clasificar de acuerdo con sus propios criterios; sin embargo el Decreto 4747 del 2007 señalo que el "triage", debería ser de obligatorio cumplimiento por todas las instituciones prestadoras de servicios que tuviesen habilitados los servicios de urgencias, al igual que de los responsables del pago de los servicios de salud (Ministerio de la Protección Social, 2007); es así como el concepto ha 
variado sustancialmente con la entrada en vigencia de la Resolución 5596 de 2015, por la cual se definen los criterios técnicos para el sistema de selección y clasificación de pacientes en los servicios de urgencias. El artículo 3 de esta norma lo define como:

El triage en los servicios de urgencia es un Sistema de Selección y Clasificación de pacientes, basado en sus necesidades terapéuticas y los recursos disponibles que consiste en una valoración clínica breve que determina la prioridad en que un paciente será atendido. El "Triage", como proceso dinámico que es, cambia tan rápidamente como lo puede hacer el estado clínico del paciente" (Ministerio de Salud y Protección Social, 2015).

El alcance de la Resolución va más allá de la definición y establece quiénes son los responsables de llevar a cabo la actividad, fija los criterios para establecer la gravedad de los pacientes y da orientaciones básicas para la toma de decisiones clínicas.

La circular externa 014 de 1995 de la Superintendencia Nacional de Salud plantea que la atención inicial de urgencias no podrá estar condicionada por garantía alguna de pagos posterior ni afiliación previa, y que es el médico quien define esta condición (Superintendencia Nacional de Salud, 1995). La jurisprudencia también ha hecho referencia en cuanto al servicio de urgencias y ha aclarado la conexidad existente entre el derecho a la salud con el derecho a la vida "la afectación del derecho a la vida no puede ser entendida sólo cuando la persona está a punto de morir, sino que el concepto es más amplio, se extiende hasta el punto de garantizar una existencia en condiciones dignas" (Sentencia T-790 de 2013).

En la actualidad, la accesibilidad y la oportunidad de la atención en las salas de urgencias se han visto seriamente afectadas debido a la congestión creciente en estas áreas hospitalarias que ocasiona demoras en la prestación de los servicios, negación de la atención, quejas e incomodidades y en algunos casos, lo que se ha denominado el "paseo de la muerte" o de manera análoga el "tren de la muerte".

Dicha expresión hace referencia a la ocurrencia de la muerte de los pacientes que no logran recibir la atención requerida, a causa de la contención de costos, las barreras de acceso interpuestas por las instituciones, la negación total o parcial de servicios del plan obligatorio, la demora en la expedición de autorizaciones o servicios por motivos administrativos, ocasionando el desenlace fatal y reiterando la ineficacia del Sistema de Seguridad Social para garantizar el derecho fundamental de la salud (Gañan, 2010).

Además de los anteriores factores, la crisis que hoy registran las salas de urgencias ha sido ocasionada por la falta de oportunidad en la atención médica por consulta externa, la falla en el seguimiento y control en los programas de promoción de la salud y prevención de la enfermedad y los factores culturales de los usuarios que han motivado una afluencia desmedida de pacientes que supera la capacidad de respuesta de estas áreas en los aspectos humanos, técnicos y físicos.

El tema de las demoras y la congestión en urgencias, ha suscitado un intenso debate que pone a prueba la capacidad de respuesta de las instituciones y los profesionales de salud. La atención en las salas de urgencias debe ser inmediata, oportuna y efectiva para suplir las necesidades físicas y psicológicas de los pacientes en situaciones que suponen una amenaza para la vida, hecho que en la mayoría de los casos no se cumple, a pesar de que ya haya entrado en vigor la Ley Estatutaria en Salud y que posteriormente se hayan creado normas subsidiarias, como la norma que establece el triage.

En nuestro país el servicio de urgencias cada vez es más preocupante, porque se ha 
incrementado la demanda y esto a raíz de un modelo en donde los servicios de salud electivos e incluso la atención que hace parte de los planes de beneficios vigentes son negados de manera sistemática y existen barreras claras en cuanto a la accesibilidad y oportunidad de estos, es así como los usuarios han encontrado una "vía alterna" para acceder de una manera rápida y eficaz a esos servicios que se le debieron haber prestado de manera ambulatoria, congestionando así los servicios de urgencias y demandando capacidad instalada como recursos humanos, económicos, tecnológicos y físicos que podrían utilizarse en la atención de urgencias reales; también impacta al sistema a nivel general ya que servicios que se encontraban capitados previamente, deben ser cancelados por evento (al considerarse urgente) (Arroyave, 2007).

Debido a la normatividad vigente que busca blindar los servicios de urgencias para que cumplan criterios de accesibilidad y oportunidad, muchas instituciones de salud han implementado prácticas que vulneran el principio de continuidad en salud, ya que brindan la atención inicial o la estabilización para no incumplir la norma, pero no continúan el tratamiento requerido por la condición del paciente, generando secuelas, invalideces y discapacidades e incluso la muerte.

La realidad cotidiana de los servicios de salud, muestra un horizonte totalmente diferente que se refleja en la demora de la atención y la saturación de las salas de urgencias, pues los protocolos establecidos al interior y entre los prestadores, suponen la realización de procesos administrativos que se han ido configurando como barreras de acceso a los servicios de salud.

\section{Los nuevos retos del derecho fundamental a la salud}

La ley estatutaria definió unos principios y elementos esenciales que deben regir el sistema y la prestación de los servicios con el fin de garantizar que efectivamente el aspecto relacionado con uno de los determinantes de la salud que es el sistema sanitario, se convierta en un factor que contribuya al mejoramiento de esta y ayude a alcanzar el mayor grado posible de salud de la población Colombiana; así mismo abarca unos aspectos importantes relacionados con la prestación de los servicios de salud y hace puntualidad en varios aspectos que a la fecha se habían detectado problemáticos y que pretenden garantizar el goce efectivo del derecho.

Para que estos cambios se conviertan en una realidad de cara al paciente, al usuario del sistema, se deben dar una serie de cambios operativos, administrativos, económicos, sociales y laborales que finalmente impactarán el momento de verdad del usuario del sistema; algunos de estos cambios obligan a que las entidades promotoras de salud tengan que repensar e implementar cambios en la forma de hacer las cosas ya que las medidas adoptadas hasta el momento no han reportado una mejoría significativa, lo que muestra la necesidad de adoptar nuevas decisiones con miras a garantizar el goce efectivo del derecho a la salud de los Colombianos.

\section{Los Planes de beneficios con la nueva Ley}

En cuanto al plan de beneficios la Ley Estatutaria de Salud, diseña un cambio profundo, pues el listado actual que conforma dicho plan o generalmente Ilamado Plan Obligatorio de Salud (POS), el cual define de manera explícita los medicamentos, procedimientos e insumos a los que los afiliados del sistema de salud tiene acceso, desaparecerá y será un plan de beneficios basado en un régimen de exclusión.

Este cambio se previó para un periodo de transición de dos años a partir de la expedición de la Ley, el Gobierno Nacional y el Congreso de la República deberán establecer las políticas para la definición de un nuevo esquema 
de beneficios, incluido el mecanismo para realizar las exclusiones según los criterios establecidos por la misma ley, en el lapso de tiempo que tomara esta implementación se seguirán aplicando las reglas vigentes en el sistema de salud; por esa razón recientemente el Ministerio de la protección social expidió la resolución 5592 de 2015 (Ministerio de Salud y Protección Social, 2015) con el fin de resolver en este periodo de transición la falta de actualización del plan de beneficios conocido como POS y algunos mecanismos usados en dicha actualización.

Es decir, que los afiliados al sistema de salud tendrán derecho a todo lo que los médicos les ordenen, excepto a algunos elementos que, de manera explícita, quedan por fuera del régimen.

Al distanciarnos de un plan de beneficios limitado que detalla puntualmente su cobertura, le damos paso al racionamiento implícito, introduciendo una lista negativa de servicios que no se financiarán, pero que en casos muy excepcionales estas exclusiones podrán ser solicitadas a través de un proceso individual que será definido y estudiado por las juntas médicas específicas.

Estas son las exclusiones definidas en la ley estatutaria en su artículo 15 (Ley 1751 de 2015) para el plan de beneficios:

- Lo cosmético y lo suntuario.

- Lo experimental.

- Lo que no tenga evidencia científica.

- Los tratamientos en el exterior que puedan ser brindados en el país.

En principio, bajo el supuesto de que lo excluido abarca un universo menor que lo implícitamente incluido, una lista negativa sería más fácil de elaborar que una lista positiva, ya que se entiende que todo el contenido de un listado positivo ha sido evaluado de alguna manera
(Giedion, Bitran \& Tristao, 2014); de tal manera elaborar una lista positiva puede requerir un trabajo intenso, ya que se debe evaluar cada tecnología y cada vez que se quiere añadir una nueva tecnología (Giedion, Bitran \& Tristao, 2014).

En este caso con la nueva ley a nivel de la provisión de los servicios de salud, una lista negativa concederá mayor autonomía a los prestadores, pues pueden decidir libremente qué tecnologías usar siempre y cuando no formen parte de la lista negativa (Giedion, et. al., 2014).

En general hay acuerdo en que los recursos, siempre escasos, no alcanzan para proveer todas las tecnologías disponibles a todos quienes las necesitan, y que la brecha entre lo médicamente posible y lo financieramente factible crece con el tiempo. Es así como inevitablemente nos vemos enfrentados al racionamiento de los servicios de salud.

Las medidas de racionamiento son soportadas en la actualidad por la continuidad de Los Comités Técnico Científicos (CTC); estos serán reemplazados, pues la Ley Estatutaria reguló de forma general e indeterminada la existencia de las juntas médicas de los prestadores de servicios de salud y las juntas médicas de la red de prestadores de servicios salud.

Estas serán las encargadas de resolver las diferencias en diagnósticos y/o alternativas terapéuticas generadas a partir de la atención. Para su funcionamiento y su relación frente a otras instancias del Sistema, estas juntas médicas deberán cumplir con las condiciones impuestas por la Corte Constitucional, principalmente en lo relacionado con su procedimiento, el cual deberá responder a los principios de oportunidad, eficiencia y acceso efectivo que caracterizan al derecho fundamental a la salud.

Es claro que diseñar los planes de beneficios no será solo una tarea para un personal 
técnico, ya que garantizar la protección del derecho fundamental a la salud tendrá un gran contenido político. En Colombia es necesario construir un marco institucional sólido donde prevalecen los procesos participativos, transparentes y sistemáticos. De lo contrario, se materializará el riesgo de que los planes de beneficios sean definidos y ajustados con criterios que responden más a la coyuntura política o los intereses de pocos, que a las verdaderas necesidades y capacidades del país; situación que mantendrá vigentes los mecanismos judiciales y excepcionales para acceder a servicios no incluidos en los planes de beneficios amenazando nuevamente la sostenibilidad y legitimidad del sistema.

\section{Autonomía médica a partir de la ley estatu- taria}

Como vemos en la actualidad colombiana el sistema de salud responde a un sistema de mercantilización de los servicios de salud; esto ha generado una gran afectación en la manera en que el médico y el paciente se relacionan; pues las decisiones no solo responden a criterios de autonomía del paciente y médico tratante, sino están sujetas a la "aprobación" del tercero (aseguradora o pagador); adicionalmente con la filosofía del mercado, de la utilidad y del hacer eficientes los recursos, el médico y los profesionales de la salud sufren presiones externas para que sus decisiones se acomoden a los presupuestos hechos y no a las necesidades reales de salud de sus pacientes, sin hablar de las figuras laborales que se usan en la actualidad para abaratar la mano de obra en salud pero que no garantizan los beneficios laborales que todo trabajador debería tener.

Por esta razón en las distintas reformas propuestas hubo un aparte específico para el ámbito de las decisiones en salud y la forma en que los galenos orientan sus tratamientos y decisiones clínicas; en cuanto a este tema la Ley Estatutaria en su Capítulo III artículo 17 habla de la autonomía del profesional de la salud y dice:
Se garantiza la autonomía de los profesionales de la salud para adoptar decisiones sobre el diagnóstico y tratamiento de los pacientes que tienen a su cargo. Esta autonomía será ejercida en el marco de esquemas de autorregulación, la ética, la racionalidad y la evidencia científica. Se prohíbe todo constreñimiento, presión o restricción del ejercicio profesional que atente contra la autonomía de los profesionales de la salud, así como cualquier abuso en el ejercicio profesional que atente contra la seguridad del paciente. La vulneración de esta disposición será sancionada por los tribunales u organismos profesionales competentes y por los organismos de inspección, vigilancia y control en el ámbito de sus competencias". (Ley 1751 de 2015)

Esta normatividad pretende mejorar la relación médico-paciente en el entendido que las autonomías aquí comprometidas son precisamente esas dos; por una parte la del médico como agente del sistema y con la capacidad técnica para orientar la decisión y por otra parte la libertad de elección del paciente para aceptar o no dicha recomendación o solicitar la posibilidad de alternativas terapéuticas, con respecto a este asunto la corte constitucional pronuncio: "el ejercicio médico se encuentra estructurado a partir de dos principios fundamentales: capacidad técnica del médico y consentimiento idóneo del paciente" (Sentencia T- 401 de 1994)

De esta forma la Ley Estatutaria pretende devolver al profesional de la salud y al paciente (principales actores en la prestación de los servicios de salud) los elementos esenciales para que su relación terapéutica cobre nuevamente importancia y las decisiones emanadas del acto médico se conviertan en una realidad y permitan el restablecimiento y/o la permanencia del estado de salud. Se espera entonces que la autonomía médica y la de los pacientes ya convertidos en una realidad permitan la recuperación de la confianza y 
concluyan en calidad y humanización de la atención médica (Calderón, 2015).

Sin embargo para ello la misma ley estatutaria recalca la importancia de la "autorregulación" y en cuanto a ello dice:

"Los conflictos o discrepancias en diagnósticos y/o alternativas terapéuticas generadas a partir de la atención, serán dirimidos por las juntas médicas de los prestadores de servicios de salud, utilizando criterios de razonabilidad científica, de acuerdo con el procedimiento que determine la ley" (Ley 1751 de 2015).

Se debe dejar claro para no restar legitimidad a los planes de beneficios no alimentar la ilusión de que todo lo clínicamente eficaz podría ser financiado y no ser excluido, y no alimentar la idea de que la autonomía médica carece de restricciones, estos mismos elementos podrían seguir atentando contra la estructuración del sistema de salud.

\section{Las Entidades Promotoras de Salud y el nuevo plan de beneficios.}

Ya que el Plan de beneficios actual cambiará y se basará en un sistema de cobertura amplio con unas exclusiones específicas, las EPS deberán hacer parte activa del desarrollo de los mecanismos técnicos, participativos y transparentes que definan los servicios y tecnologías que serán excluidas.

Para esto las EPS tienen la posibilidad de conformar un equipo técnico, médico y financiero que les permita tomar una posición frente a los servicios que no serán cubiertos y frente a las posibles tecnologías y servicios que deberán ahora garantizar a su población afiliada. Es importante que estas entidades busquen espacios de participación que les permitan tener injerencia en la definición de la UPC (la cual es obligación del estado para con estas aseguradoras) ya que cuantificar el costo de la atención de manera adecuada le permitirá al país dimensionar y apropiar los recursos necesarios para garantizar el derecho a la salud de los usuarios (Giedion, et. al., 2014).

Igualmente las aseguradoras no solo jugaran un papel fundamental en el diseño del plan de beneficios, sino que deberán preocuparse por la implementación y funcionamiento del mismo; para esto es necesario que piensen en la creación de unidades especiales que se encarguen del control financiero, de la calidad del mismo, y de la divulgación; ya que esta sería la forma idónea para que las personas puedan hacer exigible su cumplimiento y dejen de lado los mecanismos alternativos como la tutela, a la cual se le atribuye el desequilibrio económico en el que se ha sumido el sistema.

Por otra parte al tener un plan de beneficios con una lista negativa, las nuevas tecnologías y servicios que van surgiendo en teoría son automáticamente incluidas en dicho plan a menos que cumpla con los criterios de exclusión definidos por la norma; por tal motivo es importante que las EPS hagan seguimiento frecuente a las nuevas tecnologías en salud y de acuerdo con estas, sugieran los cambios apropiados al plan de beneficios (en busca de su fortalecimiento) pero a la vez proyecten los costos que traerán los mismos, para así garantizar una cobertura costo efectiva y tener elementos técnicos que les permitan negociar de una manera objetiva las UPC que incluso podrían pasar de ser de periodicidad anual a una periodicidad semestral o trimestral de acuerdo al comportamiento del plan de beneficios.

En cuanto a la autonomía médica la ley estatutaria de Salud buscó protegerla en pro de garantizar el goce efectivo del derecho a través del cumplimiento o la materialización de los procedimientos, servicios o terapias ofrecidos por el profesional de salud y aceptados por el usuario dentro del marco autonomía profesional - autonomía del paciente.

Esto indefectiblemente tendrá un impacto en la cantidad, calidad, oportunidad y oferta de los 
servicios y tecnologías en salud de cara a los usuarios; por esta razón no solo los prestadores del servicio (IPS) tendrán que adaptarse y crear mecanismos para responder a las nuevas demandas de los servicios de salud; así mismo las EPS deberán considerar mecanismos para la racionalización del uso de los servicios sin afectar tal autonomía y sin afectar él logró de los objetivos propuestos por la ley en mira de alcanzar el derecho fundamental a la Salud.

En la actualidad los Comités Técnico Científicos (CTC) son instancias que definen la idoneidad o no de una terapia prescrita por el médico tratante, pero que se encontraba por fuera del plan explícito de beneficios; dicha instancia por normatividad podía ser conformada de manera independiente por la EPS (Ministerio de la Protección Social, 2010) y tenía representación de la EPS, la IPS y 1 representante de los usuarios.

Con la Ley estatutaria y el termino Juntas Médicas (quienes ahora definirán ...los conflictos o discrepancias en diagnósticos y/o alternativas terapéuticas generadas a partir de la atención...) (Ley 1751 de 2015) deja la impresión de que sus integrantes deberán ser personas conconocimiento médicoyexperiencia profesional en el campo; y aunque aún no se ha regulado su creación, conformación, funciones y lineamientos, se espera que se dé dentro del marco de la autorregulación profesional; es decir con una visión holística de sus decisiones y no con la marcada visión económica que tenían los CTC.

En cuanto al funcionamiento de estas instancias, es claro que las EPS deberán buscar mecanismos que les permitan participar y aprovechar el conocimiento que de estas juntas se emane para retroalimentar sus sistemas de autorización, hacer más eficiente dicho proceso y evitar demoras y fallas en la oportunidad y accesibilidad a los servicios y tecnologías en Salud.
Es importante hacer claridad en que la autonomía de la que habla la ley estatutaria, no significa plenipotencialidad de los médicos; sino que busca evitar la coacción y el constreñimiento para la toma de decisiones en salud; sin embargo la costó-efectividad, la evidencia y la razonabilidad científica siguen siendo criterios básicos al momento de evaluar un servicio o tecnología en salud y si esta es o no pertinente.

La ley estatutaria en su capítulo III menciona varios aspectos importantes para reconfigurar la autonomía profesional, entre ellas el trabajo digno de los profesionales de la salud, con una remuneración adecuada y con acceso a capacitación y actualización que permita un óptimo desempeño profesional (Ley 1751 de 2015). Al ser esta una responsabilidad de todos los actores del sistema, las EPS deberán implementar sistemas de seguimiento y vigilancia de la calidad de los servicios prestados por sus IPS contratantes; dentro del seguimiento deberán velar por que las IPS contratadas en su red cumplan la normatividad vigente y futura del talento humano en salud y verificar que no existan mecanismos de coacción a las decisiones médicas; estos aspectos podrían hacer parte de indicadores de seguimiento que tengan capacidad de influir en las renovaciones de contrato y tarifas pactadas.

Por último las EPS como aseguradoras del riesgo en salud, administradoras del plan de beneficios y como tercera persona en la relación médico-paciente, deberán encontrar la forma de potenciar la prevención y promoción en esta relación terapéutica; garantizar capacitación a los profesionales para disminuir los costos mediante programas de seguimiento que eviten complicaciones y la aparición de enfermedades prevenibles o curables en sus fases tempranas; todo esto en pro de garantizar la sostenibilidad financiera y el mejoramiento continuo de los indicadores de salud de la población a su cargo.

Así mismo deberán jugar un papel activo en 
construir una experiencia histórica distinta en el campo de la Salud que según palabras el profesor Torres..."pasa por construcciones directas con la gente, desde sus necesidades y demandas, pero también desde sus saberes y experiencias. La salud implica fundamentalmente pensar en bienestar, bien vivir y no solo en consultas médicas, consumo de medicamentos y acceso a tecnología médica" (Torres, 2015). Todo esto en busca de un sistema menos morbicentrista que piense de verdad en la salud y sus determinantes y la enfermedad deje de ser el centro de nuestro sistema de seguridad social.

\section{La atención integral de urgencias.}

Continuando con el análisis de algunas de las implicaciones derivadas de la implementación de la Ley Estatutaria en Salud con relación a la atención de Urgencias.

Es pertinente señalar que en las últimas dos décadas se ha venido legislando intensamente sobre la prestación de los servicios y la atención en urgencias. En el año 2007, el Decreto 4747, en los artículos 10 y 11, dictaminó sobre los trámites administrativos requeridos para la prestación de los servicios, entre ellos: la validación de la identificación, la afiliación al Sistema de Seguridad Social y la verificación de los derechos en la base de datos provista por los encargados de los pagos y autorizaciones en las Entidades Promotoras de Salud (Ministerio de la Protección Social, 2007).

La verificación de requisitos y derechos en el sistema, ha generado unas barreras de acceso (Hernández, Rubiano \& Barona, 2015), en el sentido de que por inconsistencias en las bases de datos y desconocimiento de los contratos, se ha negado la atención urgente, sin tomar en consideración el estado de salud real de las personas.

Con la promulgación y puesta en marcha de la Ley Estatutaria se espera generar una transformación importante, pues los usuarios, podrían tener la posibilidad de recibir la atención de urgencias sin la necesidad de esperar las autorizaciones previas de las aseguradoras. Bajo esta óptica, se espera mejorar el acceso y la oportunidad a la atención y disminuir las amenazas que pongan en riesgo la vida de las personas.

La Ley propone una abolición de los trámites administrativos, tal como lo plantea el artículo 14:

Artículo 14. Prohibición de la negación de los servicios. Para acceder a servicios y tecnologías de salud, no se requerirá ningún tipo de autorización administrativa entre el prestador de servicios y la entidad que cumpla la función de gestión de servicios cuando se trate de gestión de urgencia. (Ley 1751 de 2015)

En este contexto, se pretende eliminar las barreras administrativas existentes tanto en las Instituciones Prestadoras de Servicios de Salud (IPS), y en las administradoras de los recursos, es decir que independientemente de que el usuario esté o no al día con los pagos a la EPS o que busque la atención en una institución prestadora de servicios diferente a la contratada por la aseguradora, se deberá garantizar siempre la atención oportuna. Este atributo surte efecto también para los tratamientos o medicamentos.

Así mismo, en aras de garantizar el acceso oportuno y defender el carácter fundamental de la salud como derecho, se salvaguarda la universalidad, en el sentido de abolir la negación de servicios aunque los usuarios no tengan afiliación vigente en el sistema.

En el plano de la asegurabilidad y los costos, la legislación reciente señala que la atención suministrada en los servicios de urgencias, debe ser pagada por las Entidades Promotoras de Salud a los prestadores, con base en las tarifas establecidas mediante negociación o de acuerdo al modelo de contratación establecido, 
tomando como referencias el manual tarifario del ISS, el SOAT, por evento o paquete, siempre y cuando se cumplan los criterios existentes para la clasificación de la situación como una urgencia, para lo cual, se emplea el triage bajo el fundamento legal recientemente emitido.

Otro elemento fundamental a considerar en este análisis, tiene que ver con el sistema de referencia y contrarreferencia establecido dentro de las redes de servicios. Desde la década de los años 90, se ha venido regulando al respecto. Inicialmente, el Decreto 2759 de diciembre de 1991 sentó las bases legales para desarrollar el régimen en el marco de prestación de servicios, determinando la utilización racional de recursos en torno a la atención de los pacientes con base en la complejidad de los problemas de salud y las capacidades de respuesta institucional (Ministerio de salud, 1991).

En el plano operativo, la referencia es el envío de pacientes o elementos de ayuda diagnóstica de un prestador de servicios a otro, para la atención o complementación diagnóstica, con base en las capacidades operativas y de resolución en respuesta a las necesidades de salud.

Si bien la Ley Estatutaria en Salud, no hace una declaración explícita sobre la referencia y contrarreferencia, establece las bases legales para continuar regulando jurídicamente al respecto, tanto desde la conceptualización sobre el Sistema de Salud, como desde el establecimiento de los principios fundamentales (disponibilidad, accesibilidad, universalidad, equidad, continuidad, oportunidad e integralidad) como preceptos que orientan a la garantía del derecho fundamental a la salud.

Precisamente, como una forma de optimizar la atención en urgencias, el Ministerio de Salud y Protección Social, formuló la Resolución 5596 del 24 de diciembre de 2015, mediante la que define los criterios técnico para el Sistema y Clasificación de pacientes en los servicios de urgencias, buscando con ello poner en marcha una regulación acorde con las características del contexto social, económico y político de la prestación de los servicios de salud (Ministerio de Salud y Protección Social, 2015).

La importancia de la presente resolución va más allá de la definición y establecer quienes son los profesionales responsables para realizar el triage, contempla los objetivos del mismo consignados y orientados asegurar una valoración rápida, disminuir el riesgo de muerte, complicaciones o discapacidad de los pacientes que accedan a los servicios de urgencia y brindar una comunicación con estos y sus acompañantes

Con el fin de dar prioridad en la atención de los pacientes en los servicios de urgencias, se han establecido una clasificación por categorías, donde la clasificación I y II, se deben dar cumplimiento a los tiempos establecidos en la presente resolución, las categorías III,IV y V los tiempos de atención serán definidos por las IPS. Los tiempos de espera de los pacientes deben estar publicados en un lugar visible

Con relación a los profesionales en salud encargados de la realización del proceso de clasificación están definidos por el nivel de atención así: las urgencias de alta y mediana complejidad a cargo de los profesionales en medicina y enfermería, mientras que los de baja complejidad por auxiliares de enfermería o tecnólogos en atención pre hospitalaria bajo supervisión médica.

\section{Los cambios en la atención de urgencias y las Entidades promotoras de Salud.}

La Organización Mundial de la Salud define la accesibilidad como la organización de una oferta de servicios que sea geográfica, financiera, cultural y funcionalmente alcanzable para toda la comunidad. Es por esto, que para garantizar una adecuada prestación de los servicios de urgencias con calidad y oportunidad, las EPS deberán fortalecer las redes integradas de 
servicios de salud donde se creen sinergias con otras instituciones y especialistas de tal manera que los usuarios no estén sometidos a barreras de acceso por recurso humano, tecnológico incluso geográfico; ampliar los horarios de consulta médica de tal manera que esto evite el congestionamiento del servicio de urgencias; Realizar programas de educación en salud para enseñar a la población cuando deben consultar a un servicio ; Incentivar a los especialistas y sub-especialistas para que presten sus servicios no solo en las grandes ciudades si no en la medianas y pequeñas mejorando el poder resolutivo de las instituciones y de esta manera mejorar el indicador de salud de la población.

Las EPS al tener intereses de tipo económico, podrán trabajar en la actualización de los manuales tarifarios de acuerdo al nuevo plan de beneficios y crear nuevas propuestas donde el enfoque de las mismas se base en indicadores de salud y gestión del riesgo y no en frecuencias y volúmenes de atención.

No obstante, no debemos pasar por alto el evaluar si el modelo de atención de urgencias implementado en Colombia es el adecuado y si este reviste la necesidad de realizar alguna modificación o por el contrario de adoptar un modelo diferente (Martínez, 2010) que pueda resultar más efectivo, por ejemplo, países como Canadá, Australia, Nueva Zelanda e Inglaterra los cuales tienen un modelo de urgencias que se fundamenta en una continuidad asistencial mediante la integración de los sistemas extra e intrahospitalarios. Así, la asistencia médica se inicia en la emergencia por parte del personal paramédico y continúa en los servicios de urgencias hospitalarios, donde se le practican al paciente los tratamientos que requiera.

\section{Conclusiones}

Con la ley estatutaria de salud se regula oficialmente el derecho fundamental a la salud y se vinculan unos principios y elementos esenciales que deberán regir la prestación de los servicios al interior del Sistema de seguridad social para hacer efectivo el goce de dicho derecho.

Aunque para algunos aspectos la Corte Constitucional definió un periodo de hasta 2 años para su implementación, el servicio de Urgencias en salud debe cumplir en la actualidad los principios y elementos esenciales definidos con el fin de garantizar una atención oportuna, de calidad y evitar desenlaces fatales que afecten la situación de morbi-mortalidad de la población Colombiana.

En la actualidad existen múltiples barreras que confinan el derecho a la Salud a una ilusión o a una letra muerta y para que esta realidad cambie se requiere la participación activa de cada uno de los actores del sistema incluyendo a los usuarios mediante el autocuidado y el cuidado de la salud colectiva.

Para que el derecho fundamental a la salud sea una realidad la sociedad Colombiana deberá entender la salud de una manera distinta, dentro de un marco de referencia integral, que involucre los determinantes sociales de la salud y que vire el sistema asistencial hacia un sistema que permita promocionar la salud y prevenir la enfermedad.

Las Entidades promotoras de salud como actores importantes dentro del sistema, pueden y tienen el deber de participar en la formulación de estrategias que permitan cumplir los elementos esenciales del derecho fundamental a la salud para garantizar la prestación de los servicios de una manera adecuada; pero además para garantizar que los recursos que le sean asignados se usen de la manera más eficiente sin desconocer los criterios de costoefectividad, racionalidad científica y financiera que aún continúan vigentes con la nueva ley. 


\section{Referencias}

Accesibilidad. (2015). Diccionario de la Lengua Española. Madrid. Disponible en: http://dle.rae.es/?id=0K2DI9N. Acceso el 15 de diciembre 2015.

Alegret, M., Grau Abalo, R., \& Rodríguez, M. (2008). El enfoque espacio-temporal-contextual en el estudio del VIH-SIDA. Revista Cubana De Salud Pública, 34(3). $\quad$ http://dx.doi.org/10.1590/s0864$\underline{34662008000300003 .}$.

Arroyave, I, (2007). Circular marco de urgencias. Periódico El pulso, No. 1, (107). Medellín.

Asociación Médica Mundial. (1987). Declaración de Madrid sobre la Autonomía y Autorregulación profesionales. Madrid. Disponible en: http://www.wma.net/es/3 Opublications/10policies/20archives/a21/

Calderón, J. (2015). Autonomía médica y ley estatutaria de salud. Acta Médica Colombiana, 40(1), 51 - 53.

Comes, Y., Solitario, R., Garbus, P., Mauro, M., Czerniecki, S., Vázquez, A., Sotelo, R., \& Stolkiner, A. (2007). El concepto de accesibilidad: la perspectiva relacional entre población y servicios. Anuario de investigaciones, 14, 00. Recuperado en 20 de Diciembre de 2015, de http://www.scielo.org.ar/scielo.php?script=sci arttext\&pid=S1851$16862007000100019 \&$ Ing=es\&tIng=es.

Continuidad. (2015). Diccionario Etimológico. Chile. Disponible en: http://etimologias.dechile.net/

Cuasialpud, J. (2013). LaAutonomía Médica en Colombia a partir de la Ley 100 de 1993 (Ensayo para optar al Título de Especialista en educación). Universidad Militar Nueva Granada.
De la Calle, A. (2006). Pérdida de oportunidad en salud. Ars Médica. Revista De Humanidades, 5(1), 141-147. Disponible en: https://www.fundacionpfizer.org/sites/default/files/pdf/ars medica jun 2006 num09 136142 calle.pdf

Duran, L. (2004). Calidad en la Prestación de Servicios de Salud. Revista. Universidad la Salle. Num.3, 3-7.

El Tiempo, Redacción. (2012). El 40\% de pacientes está inconforme con los servicios de salud Estudio de percepción sobre el sistema halló que siguen las demoras en asignación de citas. El Tiempo. Disponible en: http://www.eltiempo.com/archivo/ documento/CMS-12421628

Gálvez, M. (2003). Continuidad asistencial. Análisis conceptual, de los actores y amenazas. Propuestas y alternativas. Medicina De Familia, 4(1), 58. Recuperado de http://www.samfyc.es/Revista/ PDF/v4n1/09.pdf.

Gañan, J. (2013). Los muertos de la Ley 100. Medellín: Universidad de Antioquia.

Giedion, U., Bitran, R., \& Tristao, I. (2014). Planes de beneficios en salud de América Latina. Washington D.C.: Inter-American Development Bank.

Gomez, R., \& Nieto, E. (2014). Colombia: ¿qué ha pasado con su reforma de salud? Revista Peruana De Medicina Experimental Y Salud Publica, 31(4), 733-739. Disponible en http://www. scielo.org.pe/scielo.php?pid=S1726$\underline{46342014000400019 \& \text { script }=\text { sci arttext }}$

González, N. Martínez, J. Ureta, C \& Alonso, S. (2012). Protocolo de triage o Recepción, Acogida y Clasificación (RAC) de Enfermería en Urgencias del Hospital Do Salnés. 23-01-2016, Disponible en: http://www.enferurg.com/articulos/protocolorac.htm) 
Gracia, D. (1998). Profesión médica, investigación y justicia sanitaria. Bogotá, D.C.: Editorial El Búho.

Gualtero, M. (2007) Autonomía médica y su relación con la prestación de los servicios de salud. Bogotá, D.C.: Imprenta Nacional.

Hernández, J., Rubiano, D., \& Barona, J. (2015). Barreras de acceso administrativo a los servicios de salud en población Colombiana, 2013. Ciênc. Saúde Coletiva, 20(6), 19471958. http://dx.doi.org/10.1590/141381232015206.12122014

Integridad. (2015). Diccionario de la Lengua Española. Madrid. Disponible en: http:// dle.rae.es/?id=LqKu5NS. Acceso el 23 de diciembre 2015.

Kloh, D., Reibnitz, K., Boehs, A., Wosny, A., \& Lima, M. (2014). Principio de la integralidad del cuidado en los proyectos Político-Pedagógico de los cursos de enfermería. Revista Latino-Americana De Enfermagem, 22(4), 693-700. http:// dx.doi.org/10.1590/0104-1169.3381.2469

Martinez, A. \& Rubio, V.J. (1999). Enfoque biopsicosocial de la salud. Madrid: UAM (mímeo). Disponible en: http://www.uam. es/personal_pdi/psicologia/victor/SALUD/Bibliog/concepto salud.PDF

Martínez, G. (2010). Organización de los Servicios de Urgencias: Modelos organizativos. Instituto de Formación continua. Universidad de Barcelona. 1,. 1-4. Disponible en: http://cvirtual2.il3.ub.edu/repository/coursefilearea/file.php/1/mf/cursos/ sal_meuh/sal_meuh_m2t1.pdf).

Méndez Aristizábal, I. (2015). El Derecho fundamental a la salud: Repensando su efectividad en Colombia con especial atención a la salud de las mujeres (Trabajo Fin de Máster). Universidad Carlos III de Madrid.

Morales, C. (2008). Evaluación de la oportunidad en la atención en el Servicio de Urgencias en la E.S.E. Hospital San Juan de Dios de Girón octubre-diciembre de 2008 (Trabajo de grado optar título de especialista). Universidad CES.

Oportunidad. (2015). Diccionario de la Lengua Española. Madrid. Disponible en: http://dle.rae.es/?id=R6zLykN. Acceso el 23 de diciembre 2015.

Organización de las Naciones Unidas. Asamblea General. (1976). Pacto Internacional de Derechos Económicos, Sociales y Culturales. Resolución de la Asamblea General 2200 A (XXI) del 16 de diciembre de 1966. Ginebra.

Organización de las Naciones Unidas. Asamblea General. Declaración Universal de los DDHH. 1948.

Organización de los Estados Americanos. (1988). Protocolo adicional a la Convención Americana sobre Derechos Humanos en materia de Derechos Económicos, Sociales y Culturales. "Protocolo de San Salvador". San Salvador: Secretaría General OEA.

Organización Mundial de la Salud, Conceptos de urgencia y emergencia. Valoración y cuidados de enfermería ante situaciones críticas, .2010 (Citado 14/07/2015). Disponible en: http://www.grupocto.es/ tienda/pdf/EN_OPECan_CapM.pdf

República de Colombia, Corte Constitucional (1994, septiembre), "Sentencia T - 401", M. P. Cifuentes Muñoz, E., Bogotá. 
República de Colombia, Corte Constitucional (2000, Febrero), "Sentencia T - 179", M. P. Martínez Caballero, A., Bogotá.

República de Colombia, Corte Constitucional (2002, octubre), "Sentencia T - 823", M.

P. Escobar Gil, R., Bogotá.

República de Colombia, Corte Constitucional (2003, diciembre), "Sentencia T - 860", M. P. Montealegre Lynett, E., Bogotá.

República de Colombia, Corte Constitucional (2003, octubre), "Sentencia T - 988”, M. P. Monroy Cabra, M., Bogotá.

República de Colombia, Corte Constitucional (2003, Septiembre), "Sentencia T - 859", M. P. Montealegre Lynett, E., Bogotá.

República de Colombia, Corte Constitucional (2004, febrero), "Sentencia T - 136", M. P. Cepeda Espinosa, M., Bogotá.

República de Colombia, Corte Constitucional (2006, julio), "Sentencia T - 518", M. P. Monroy Cabra, M., Bogotá.

República de Colombia, Corte Constitucional (2007, enero), "Sentencia T - 016", M. P. Sierra Porto, H. A., Bogotá.

República de Colombia, Corte Constitucional (2007, julio), "Sentencia T - 568", M. P. Monroy Cabra, M., Bogotá.

República de Colombia, Corte Constitucional (2008, julio), "Sentencia T - 760", M. P. Cepeda Espinosa, M. J., Bogotá.

República de Colombia, Corte Constitucional (2008, junio), "Sentencia T - 604", M. P. Monroy Cabra, M., Bogotá.
República de Colombia, Corte Constitucional (2008, octubre), "Sentencia T - 657", M. P. Sierra Porto, H., Bogotá.

República de Colombia, Corte Constitucional (2013, abril), "Sentencia T - 211", M. P. Vargas Silva, L. E., Bogotá.

República de Colombia, Corte Constitucional (2013, abril), "Sentencia T - 214", M. P. Vargas Silva, L. E., Bogotá.

República de Colombia, Corte Constitucional (2013, noviembre), "Sentencia T - 790", M. P. Guerrero Perez, L., Bogotá.

República de Colombia, Corte Constitucional (2013, octubre), "Sentencia T - 745", M. P. Pretelt Chaljub. J.I., Bogotá.

República de Colombia, Corte Constitucional (2015, marzo), "Sentencia T - 121", M. P. Guerrero Pérez, L. G., Bogotá.

República de Colombia, Corte Constitucional (206, enero), "Sentencia T - 025", M. P. Beltrán Sierra. A., Bogotá.

República de Colombia, Ministerio de Salud y Protección Social. (2015). Atributos de la Calidad en la Atención en Salud. Minsalud.gov.co. Disponible 20 diciembre 2015 en: https://www.minsalud.gov. co/salud/Paginas/ATRIBUTOS-DE-LACALIDAD-EN-LA-ATENCI\%C3\%93NEN-SALUD.aspx

República de Colombia. Congreso de la Republica. Ley 10 de 1990. (10, enero, 1990). Por la cual se reorganiza el Sistema Nacional de Salud y se dictan otras disposiciones. 
República de Colombia. Congreso de la Republica. Ley 10 de 1990. Artículo 2. (10, enero, 1990). Por la cual se reorganiza el Sistema Nacional de Salud y se dictan otras disposiciones.

República de Colombia. Congreso de la República. Ley 100, Artículo 153. Numeral 3. (23, diciembre, 1993). Por la cual se crea el Sistema de Seguridad Social y se dictan otras disposiciones.

República de Colombia. Congreso de la República. Ley 100, Articulo 162. (23, diciembre, 1993). Por la cual se crea el Sistema de Seguridad Social y se dictan otras disposiciones.

República de Colombia. Congreso de la República. Ley 100, Articulo 2. (23, diciembre, 1993). Por la cual se crea el Sistema de Seguridad Social y se dictan otras disposiciones.

República de Colombia. Congreso de la República. Ley 100. (23, diciembre, 1993). Por la cual se crea el Sistema de Seguridad Social y se dictan otras disposiciones.

República de Colombia. Congreso de la República. Ley 1438. (19, Enero, 2011). Por medio de la cual se reforma el Sistema General de Seguridad Social y se dictan otras disposiciones.

República de Colombia. Congreso de la República. Ley 1438. Artículo 25. (19, Enero, 2011). Por medio de la cual se reforma el Sistema General de Seguridad Social y se dictan otras disposiciones.

República de Colombia. Congreso de la República. Ley 1751. (16, febrero, 2015). Por la cual se regula el derecho fundamental a la Salud.
República de Colombia. Congreso de la República. Ley 1751. Artículo 14. (16, febrero, 2015). Por la cual se regula el derecho fundamental a la Salud.

República de Colombia. Congreso de la República. Ley 1751. Artículo 15 (16, febrero, 2015). Por la cual se regula el derecho fundamental a la Salud.

República de Colombia. Congreso de la República. Ley 1751. Capitulo III (16, febrero, 2015). Por la cual se regula el derecho fundamental a la Salud.

República de Colombia. Congreso de la República. Ley 1751. Capitulo III Artículo 8. (16, Febrero, 2015). Por medio de la cual se regula el Derecho Fundamental a la salud y se dictan otras disposiciones.

República de Colombia. Congreso de la República. Ley 1751. Capitulo III Artículo 17. (16, Febrero, 2015). Por medio de la cual se regula el Derecho Fundamental a la salud y se dictan otras disposiciones.

República de Colombia. Congreso de la República. Ley 23. (18, febrero, 1981). Por la cual se dictan normas en materia de ética médica.

República de Colombia. Ministerio de la Protección Social. Resolución 548. Articulo 1 (12, febrero, 2010). Por la cual se reglamentan los Comités Técnico-Científicos, se establece el procedimiento de radicación, reconocimiento y pago de recobros ante el Fondo de Solidaridad y Garantía -Fosyga- y se dictan otras disposiciones aplicables durante el período de transición de que trata el artículo 19 del Decreto Legislativo 128 de 2010.

República de Colombia. Ministerio de Salud y Protección Social. Decreto 131. (21, enero, 2010). Por medio del cual se crea el 
Sistema Técnico Científico en Salud, se regula la autonomía profesional y se definen aspectos del aseguramiento del Plan Obligatorio de Salud y se dictan otras disposiciones.

República de Colombia. Ministerio de Salud y Protección Social. Decreto 2174. (28, noviembre, 1996). Por el cual se organiza el Sistema Obligatorio de Garantía de Calidad del Sistema General de Seguridad Social en Salud.

República de Colombia. Ministerio de Salud y Protección Social. Decreto 3045. (27, diciembre, 2013). Por el cual se establecen unas medidas para garantizar la continuidad en el aseguramiento y se dictan otras disposiciones.

República de Colombia. Ministerio de Salud y Protección Social. Resolución 5592. (24, diciembre, 2015). Por la cual se actualiza integralmente el Plan de Beneficios en Salud con cargo a la Unidad de Pago por Capitación-UPC del Sistema General de Seguridad Social en Salud -SGSSS y se dictan otras disposiciones.

República de Colombia. Ministerio de Salud y Protección Social. Resolución 5596. Articulo 3 (24, Diciembre, 2015). Por la cual se definen los criterios técnicos para el Sistema de Selección y Clasificación de pacientes en los servicios de urgencias "Triage"

República de Colombia. Ministerio de Salud. Decreto 2759. (11, diciembre, 1991). Por el cual se organiza y establece el régimen de referencia y contra referencia.

República de Colombia. Ministerio de Salud. Resolución 13437. (01, Noviembre, 1991). Por la cual se constituyen los comités de Ética Hospitalaria y se adopta el Decálogo de los Derechos de los Pacientes.

República de Colombia. Ministerio la Protección Social. Decreto 4747. (7, Diciembre, 2007). Por medio del cual se regulan algunos aspectos de las relaciones entre los prestadores de servicios de salud y las entidades responsables del pago de los servicios de salud de la población a su cargo, y se dictan otras disposiciones.

República de Colombia. Ministerio de Salud y Protección Social de Colombia. Resolución 5596. (24, Diciembre, 2015). Por la cual se definen los criterios técnicos para el Sistema de Selección y Clasificación de pacientes en los servicios de urgencias "Triage".

República de Colombia. República de Colombia. Constitución Política de Colombia 1991. PREAMBULO (20, Julio ,1991).

República de Colombia. Superintendencia Nacional de Salud. Circular externa 014. (28, Diciembre, 1995). Por el cual se imparten instrucciones que permitan garantizar el acceso a la atención inicial de urgencias y a la atención de urgencias, así como orientar al sector salud sobre la forma de garantizar la financiación de este tipo de atención.

República de Colombia. Constitución política de Colombia [Const.] (1991) 2da Ed. Legis.

Secretaría Distrital de Salud de Bogotá. Resolución 249 (20, Marzo, 1998). Por la cual se orienta el cumplimiento y se fija el sistema de coordinación de los Servicios de Urgencias y se dictan otras disposiciones en Santa fe de Bogotá, D.C.

Semana. Redacción. (2014). Así controlan las instituciones y empresas de salud a los médicos. Semana. Disponible en: 
http://www.semana.com/nacion/articulo/ las-eps-controlan-los-medicos-con-polemicos-metodos/409528-3

Superintendencia Nacional de Salud. (2015). Monitor Estratégico. Una herramienta para la Inspección, Vigilancia y control (pp. 4-5). Bogotá D.C.: Claudia Vásquez.

Torres, M. (2015). La ley estatutaria en salud: Una oportunidad para concretar la realización del derecho a la salud en Colombia. Semanario Virtual Caja de Herramientas. Disponible en: http://viva.org.co/ cajavirtual/svc0438/articulo05.html
Tovar-Cuevas, L., \& Arrivillaga-Quintero, M. (2014). Estado del arte de la investigación en acceso a los servicios de salud en Colombia, 2000-2013: revisión sistemática crítica. Gerencia Y Políticas De Salud, 13(27). http://dx.doi.org/10.11144/ javeriana.rgyps13-27.eaia

Urgencia. (2015). Diccionario de la Lengua Española. Madrid. Disponible en: http:// dle.rae.es/?id=b9PI6ly Acceso el 23 de diciembre 2015.

Vélez, A. (2012). Salud y Enfermedad: La contribución de la Corte Constitucional de Colombia. Revista Hacia la Promoción de la Salud, 34 (3). 91-109 Universidad de Caldas.

Forma de citar: Ramírez, A., Rocha, D., Durango, L., Rodríguez, S. (2016). Implicaciones de la ley estatutaria 1751 de 2015 sobre las entidades promotoras de salud del régimen contributivo. Rev. CES Derecho, 7(2), $13-41$. 